\title{
Nontrivial solutions for a higher fractional differential equation with fractional multi-point boundary conditions
}

\author{
Min Jia ${ }^{1 *}$, Xinguang Zhang ${ }^{2 *}$ and Xuemai Gu ${ }^{1}$
}

\section{"Correspondence:}

jiamin@hit.edu.cn;

zxg123242@sohu.com

'Communication Research Center,

Harbin Institute of Technology,

Harbin 150080, China

${ }^{2} \mathrm{~S}$ chool of Mathematical and

Informational Sciences, Yantai

University, Yantai 264005, China

\begin{abstract}
This paper investigates the existence and uniqueness of nontrivial solutions to a class of fractional nonlocal multi-point boundary value problems of higher order fractional differential equation, this kind of problems arise from viscoelasticity, electrochemistry control, porous media, electromagnetic and signal processing of wireless communication system. Some sufficient conditions for the existence and uniqueness of nontrivial solutions are established under certain suitable growth conditions, our proof is based on Leray-Schauder nonlinear alternative and Schauder fixed point theorem.
\end{abstract}

MSC: 34B15; 34B25

Keywords: fractional differential equation; nontrivial solution; Green function; Leray-Schauder nonlinear alternative

\section{Introduction}

The purpose of this paper is to establish the existence and uniqueness of nontrivial solutions to the following higher fractional differential equation:

$$
\left\{\begin{array}{l}
-\mathscr{D}^{\alpha} x(t)=f\left(t, x(t), D^{\mu_{1}} x(t), \mathscr{D}^{\mu_{2}} x(t), \ldots, \mathbb{D}^{\mu_{n-1}} x(t)\right), \quad 0<t<1, \\
x(0)=0, \quad \mathfrak{D}^{\mu_{i}} x(0)=0, \quad \mathfrak{D}^{\mu} x(1)=\sum_{j=1}^{p-2} a_{j} \mathscr{D}^{\mu} x\left(\xi_{j}\right), \quad 1 \leq i \leq n-1,
\end{array}\right.
$$

where $n \geq 3, n \in \mathbb{N}, n-1<\alpha \leq n, n-l-1<\alpha-\mu_{l}<n-l$, for $l=1,2, \ldots, n-2$, and $\mu-\mu_{n-1}>$ $0, \alpha-\mu_{n-1} \leq 2, \alpha-\mu>1, a_{j} \in[0,+\infty), 0<\xi_{1}<\xi_{2}<\cdots<\xi_{p-2}<1, \sum_{j=1}^{p-2} a_{j} \xi_{j}^{\alpha-\mu-1} \neq 1, \mathfrak{D}^{\alpha}$ is the standard Riemann-Liouville derivative, and $f:[0,1] \times \mathbb{R}^{n} \rightarrow \mathbb{R}$ is continuous.

Differential equations of fractional order occur more frequently in different research areas such as engineering, physics, chemistry, economics, etc. Indeed, we can find numerous applications in viscoelasticity, electrochemistry control, porous media, electromagnetic and signal processing of wireless communication system, etc. [1-6].

For an extensive collection of results about this type of equations, we refer the reader to the monograph by Kilbas et al. [7], Miller and Ross [8], Podlubny [9], the papers [10-24] and the references therein.

Recently, Salem [10] has investigated the existence of Pseudo solutions for the nonlinear $m$-point boundary value problem of a fractional type. In particular, he considered the 
following boundary value problem:

$$
\left\{\begin{array}{l}
\mathfrak{D}^{\alpha} x(t)+q(t) f(t, x(t))=0, \quad 0<t<1, \alpha \in(n-1, n], n \geq 2, \\
x(0)=x^{\prime}(0)=x^{\prime \prime}(0)=\cdots=x^{(n-2)}(0)=0, \quad x(1)=\sum_{i=1}^{m-2} \xi_{i} x\left(\eta_{i}\right),
\end{array}\right.
$$

where $x$ takes values in a reflexive Banach space $E, 0<\eta_{1}<\eta_{2}<\cdots<\eta_{m-2}<1$ and $\xi_{i}>$ 0 with $\sum_{j=1}^{m-2} \xi_{j} \eta_{j}^{\alpha-1}<1 . x^{(k)}$ denotes the $k$ th Pseudo-derivative of $x$ and $\mathscr{D}^{\alpha}$ denotes the Pseudo fractional differential operator of order $\alpha$. By means of the fixed point theorem attributed to O'Regan, a criterion was established for the existence of at least one Pseudo solution for the problem (1.2).

More recently, Zhang [11] has considered the following problem whose nonlinear term and boundary condition contain integer order derivatives of unknown functions:

$$
\left\{\begin{array}{l}
\mathfrak{D}^{\alpha} x(t)+q(t) f\left(x, x^{\prime}, \ldots, x^{(n-2)}\right)=0, \quad 0<t<1, n-1<\alpha \leq n, \\
x(0)=x^{\prime}(0)=\cdots=x^{(n-2)}(0)=x^{(n-2)}(1)=0,
\end{array}\right.
$$

where $\mathbb{D}^{\alpha}$ is the standard Riemann-Liouville fractional derivative of order $\alpha, q$ may be singular at $t=0$ and $f$ may be singular at $x=0, x^{\prime}=0, \ldots, x^{(n-2)}=0$. By using the fixed point theorem of a mixed monotone operator, a unique existence result of positive solution to the problem (1.3) was established. And then, Goodrich [12] was concerned with a partial extension of the problem (1.3) by extending boundary conditions

$$
\left\{\begin{array}{l}
-D^{\alpha} x(t)=f(t, x(t)), \quad 0<t<1, n-1<\alpha \leq n, n>3, \\
x^{(i)}(0)=0, \quad 0 \leq i \leq n-2, \quad \mathbb{D}^{\alpha} x(1)=0, \quad 1 \leq \alpha \leq n-2 .
\end{array}\right.
$$

The author derived the Green's function for the problem (1.4) and showed that it satisfies certain properties. Then, by using cone theoretic techniques, a general existence theorem for (1.4) was obtained when $f(t, x)$ satisfies some growth conditions.

In recent work [13], Rehman and Khan have investigated the multi-point boundary value problems for fractional differential equations of the form

$$
\left\{\begin{array}{l}
\mathfrak{D}^{\alpha} y(t)=f\left(t, y(t), \mathbb{D}^{\beta} y(t)\right), \quad t \in(0,1) \\
y(0)=0, \quad \mathbb{D}^{\beta} y(1)-\sum_{i=1}^{m-2} \zeta_{i} \boldsymbol{D}^{\beta} y\left(\xi_{i}\right)=y_{0}
\end{array}\right.
$$

where $1<\alpha \leq 2,0<\beta<1,0<\xi_{i}<1, \zeta_{i} \in[0,+\infty)$ with $\sum_{i=1}^{m-2} \zeta_{i} \xi_{i}^{\alpha-\beta-1}<1$. By using the Schauder fixed point theorem and the contraction mapping principle, the authors established the existence and uniqueness of nontrivial solutions for BVP (1.5) provided that the nonlinear function $f:[0,1] \times \mathbb{R} \times \mathbb{R}$ is continuous and satisfies certain growth conditions. However, Rehman and Khan only considered the case $1<\alpha \leq 2$ and the case of the nonlinear term $f$ was not considered comprehensively.

Notice that the results dealing with the existence and uniqueness of solution for multipoint boundary value problems of fractional order differential equations are relatively scarce when the nonlinear term $f$ and the boundary conditions all involve fractional 
derivatives of unknown functions. Thus, the aim of this paper is to establish the existence and uniqueness of nontrivial solutions for the higher nonlocal fractional differential equations (1.1) where nonlinear term $f$ and the boundary conditions all involve fractional derivatives of unknown functions. In our study, the proof is based on the reduced order method as in [11] and the main tool is the Leray-Schauder nonlinear alternative and the Schauder fixed point theorem.

\section{Basic definitions and preliminaries}

Definition 2.1 A function $x$ is said to be a solution of BVP (1.1) if $x \in C[0,1]$ and satisfies BVP (1.1). In addition, $x$ is said to be a nontrivial solution if $x \neq \equiv 0$ for $t \in(0,1)$ and $x$ is solution of BVP (1.1).

For the convenience of the reader, we present some definitions, lemmas, and basic results that will be used later. These and other related results and their proofs can be found, for example, in [6-9].

Definition 2.2 (see [8]) Let $\alpha>0$ with $\alpha \in \mathbb{R}$. Suppose that $x:[a, \infty) \rightarrow \mathbb{R}$ then the $\alpha$ th Riemann-Liouville fractional integral is defined by

$$
I^{\alpha} x(t)=\frac{1}{\Gamma(\alpha)} \int_{a}^{t}(t-s)^{\alpha-1} x(s) d s
$$

whenever the right-hand side is defined. Similarly, with $\alpha>0$ with $\alpha \in \mathbb{R}$, we define the $\alpha$ th Riemann-Liouville fractional derivative to be

$$
\mathbb{D}^{\alpha} x(t)=\frac{1}{\Gamma(n-\alpha)}\left(\frac{d}{d t}\right)^{(n)} \int_{a}^{t}(t-s)^{n-\alpha-1} x(s) d s,
$$

where $n \in \mathbb{N}$ is the unique positive integer satisfying $n-1 \leq \alpha<n$ and $t>a$.

Remark 2.1 If $x, y:(0,+\infty) \rightarrow \mathbb{R}$ with order $\alpha>0$, then

$$
\mathfrak{D}^{\alpha}(x(t)+y(t))=\mathfrak{D}^{\alpha} x(t)+\mathfrak{D}^{\alpha} y(t) .
$$

Lemma 2.1 (see [7])

(1) If $x \in L^{1}(0,1), \rho>\sigma>0$, then

$$
I^{\rho} I^{\sigma} x(t)=I^{\rho+\sigma} x(t), \quad \mathscr{D}^{\sigma} I^{\rho} x(t)=I^{\rho-\sigma} x(t), \quad D^{\sigma} I^{\sigma} x(t)=x(t) .
$$

(2) If $\rho>0, v>0$, then

$$
\mathfrak{D}^{\rho} t^{\nu-1}=\frac{\Gamma(\nu)}{\Gamma(\nu-\rho)} t^{\nu-\rho-1} .
$$

Lemma 2.2 (see [8]) Assume that $x \in C(0,1) \cap L^{1}(0,1)$ with a fractional derivative of order $\alpha>0$, then $I^{\alpha} \mathfrak{D}^{\alpha} x(t)=x(t)+c_{1} t^{\alpha-1}+c_{2} t^{\alpha-2}+\cdots+c_{n} t^{\alpha-n}$, where $c_{i} \in \mathbb{R}, i=1,2, \ldots, n(n=$ $[\alpha]+1)$. Here $I^{\alpha}$ stands for the standard Riemann-Liouville fractional integral of order $\alpha>0$ and $\mathfrak{D}^{\alpha}$ denotes the Riemann-Liouville fractional derivative as Definition 2.1. 
Lemma 2.3 If $1<\alpha-\mu_{n-1} \leq 2, \alpha-\mu>1$ and $h \in L^{1}[0,1]$, then the boundary value problem

$$
\left\{\begin{array}{l}
-\mathbb{D}^{\alpha-\mu_{n-1}} w(t)=h(t), \\
w(0)=0, \quad \mathbb{D}^{\mu-\mu_{n-1}} w(1)=\sum_{j=1}^{p-2} a_{j} \mathbb{D}^{\mu-\mu_{n-1}} w\left(\xi_{j}\right)
\end{array}\right.
$$

has the unique solution

$$
w(t)=\int_{0}^{1} K(t, s) h(s) d s
$$

where $K(t, s)$ is the Green function of BVP $(2.1)$, and

$$
\begin{aligned}
& K(t, s)=k_{1}(t, s)+\frac{t^{\alpha-\mu_{n-1}-1}}{1-\sum_{j=1}^{p-2} a_{j} \xi_{j}^{\alpha-\mu-1}} \sum_{j=1}^{p-2} a_{j} k_{2}\left(\xi_{j}, s\right), \\
& k_{1}(t, s)= \begin{cases}\frac{t^{\alpha-\mu_{n-1}-1}(1-s)^{\alpha-\mu-1}-(t-s)^{\alpha-\mu_{n-1}-1}}{\Gamma\left(\alpha-\mu_{n-1}\right)}, & 0 \leq s \leq t \leq 1, \\
\frac{t^{\alpha-\mu_{n-1}-1}(1-s)^{\alpha-\mu-1}}{\Gamma\left(\alpha-\mu_{n-1}\right)}, & 0 \leq t \leq s \leq 1,\end{cases} \\
& k_{2}(t, s)= \begin{cases}\frac{(t(1-s))^{\alpha-\mu-1}-(t-s)^{\alpha-\mu-1}}{\Gamma\left(\alpha-\mu_{n-1}\right)}, & 0 \leq s \leq t \leq 1, \\
\frac{(t(1-s))^{\alpha-\mu-1}}{\Gamma\left(\alpha-\mu_{n-1}\right)}, & 0 \leq t \leq s \leq 1 .\end{cases}
\end{aligned}
$$

Proof By applying Lemma 2.2, we may reduce (2.1) to an equivalent integral equation

$$
w(t)=-I^{\alpha-\mu_{n-1}} h(t)+c_{1} t^{\alpha-\mu_{n-1}-1}+c_{2} t^{\alpha-\mu_{n-1}-2}, \quad c_{1}, c_{2} \in \mathbb{R} .
$$

Note that $w(0)=0$ and (2.4), we have $c_{2}=0$. Consequently, a general solution of (2.3) is

$$
w(t)=-I^{\alpha-\mu_{n-1}} h(t)+c_{1} t^{\alpha-\mu_{n-1}-1} .
$$

By (2.5) and Lemma 2.1, we have

$$
\begin{aligned}
\mathscr{D}^{\mu-\mu_{n-1}} w(t) & =-D^{\mu-\mu_{n-1}} I^{\alpha-\mu_{n-1}} h(t)+c_{1} D^{\mu-\mu_{n-1}} t^{\alpha-\mu_{n-1}-1} \\
& =-I^{\alpha-\mu} h(t)+c_{1} \frac{\Gamma\left(\alpha-\mu_{n-1}\right)}{\Gamma(\alpha-\mu)} t^{\alpha-\mu-1} \\
& =-\int_{0}^{t} \frac{(t-s)^{\alpha-\mu-1}}{\Gamma(\alpha-\mu)} h(s) d s+c_{1} \frac{\Gamma\left(\alpha-\mu_{n-1}\right)}{\Gamma(\alpha-\mu)} t^{\alpha-\mu-1} .
\end{aligned}
$$

So, from (2.6), we have

$$
\begin{aligned}
& \mathscr{D}^{\mu-\mu_{n-1}} w(1)=-\int_{0}^{1} \frac{(1-s)^{\alpha-\mu-1}}{\Gamma(\alpha-\mu)} h(s) d s+c_{1} \frac{\Gamma\left(\alpha-\mu_{n-1}\right)}{\Gamma(\alpha-\mu)} \\
& \mathscr{D}^{\mu-\mu_{n-1}} w\left(\xi_{j}\right)=-\int_{0}^{\xi_{j}} \frac{\left(\xi_{j}-s\right)^{\alpha-\mu-1}}{\Gamma(\alpha-\mu)} h(s) d s+c_{1} \frac{\Gamma\left(\alpha-\mu_{n-1}\right)}{\Gamma(\alpha-\mu)} \xi_{j}^{\alpha-\mu-1}, \\
& \quad \text { for } j=1,2, \ldots, p-2 .
\end{aligned}
$$


By $\mathfrak{D}^{\mu-\mu_{n-1}} w(1)=\sum_{j=1}^{p-2} a_{j} \mathfrak{D}^{\mu-\mu_{n-1}} w\left(\xi_{j}\right)$, combining with (2.7), we obtain

$$
c_{1}=\frac{\int_{0}^{1}(1-s)^{\alpha-\mu-1} h(s) d s-\sum_{j=1}^{p-2} a_{j} \int_{0}^{\xi_{j}}\left(\xi_{j}-s\right)^{\alpha-\mu-1} h(s) d s}{\Gamma\left(\alpha-\mu_{n-1}\right)\left(1-\sum_{j=1}^{p-2} a_{j} \xi_{j}^{\alpha-\mu-1}\right)} .
$$

So, substituting $c_{1}$ into (2.5), the unique solution of the problem (2.1) is

$$
\begin{aligned}
& w(t)=-\int_{0}^{t} \frac{(t-s)^{\alpha-\mu_{n-1}-1}}{\Gamma\left(\alpha-\mu_{n-1}\right)} h(s) d s+\frac{t^{\alpha-\mu_{n-1}-1}}{1-\sum_{j=1}^{p-2} a_{j} \xi_{j}^{\alpha-\mu-1}} \\
& \times\left\{\int_{0}^{1} \frac{(1-s)^{\alpha-\mu-1}}{\Gamma\left(\alpha-\mu_{n-1}\right)} h(s) d s-\sum_{j=1}^{p-2} a_{j} \int_{0}^{\xi_{j}} \frac{\left(\xi_{j}-s\right)^{\alpha-\mu-1}}{\Gamma\left(\alpha-\mu_{n-1}\right)} h(s) d s\right\} \\
& =-\int_{0}^{t} \frac{(t-s)^{\alpha-\mu_{n-1}-1}}{\Gamma\left(\alpha-\mu_{n-1}\right)} h(s) d s+\frac{\left(1-\sum_{j=1}^{p-2} a_{j} \xi_{j}^{\alpha-\mu-1}+\sum_{j=1}^{p-2} a_{j} \xi_{j}^{\alpha-\mu-1}\right) t^{\alpha-\mu_{n-1}-1}}{1-\sum_{j=1}^{p-2} a_{j} \xi_{j}^{\alpha-\mu-1}} \\
& \times \int_{0}^{1} \frac{(1-s)^{\alpha-\mu-1}}{\Gamma\left(\alpha-\mu_{n-1}\right)} h(s) d s-\frac{t^{\alpha-\mu_{n-1}-1}}{1-\sum_{j=1}^{p-2} a_{j} \xi_{j}^{\alpha-\mu-1}} \sum_{j=1}^{p-2} a_{j} \int_{0}^{\xi_{j}} \frac{\left(\xi_{j}-s\right)^{\alpha-\mu-1}}{\Gamma\left(\alpha-\mu_{n-1}\right)} h(s) d s \\
& =-\int_{0}^{t} \frac{(t-s)^{\alpha-\mu_{n-1}-1}}{\Gamma\left(\alpha-\mu_{n-1}\right)} h(s) d s+\int_{0}^{1} \frac{(1-s)^{\alpha-\mu-1} t^{\alpha-\mu_{n-1}-1}}{\Gamma\left(\alpha-\mu_{n-1}\right)} h(s) d s \\
& +\frac{t^{\alpha-\mu_{n-1}-1}}{1-\sum_{j=1}^{p-2} a_{j} \xi_{j}^{\alpha-\mu-1}} \sum_{j=1}^{p-2} a_{j} \int_{0}^{1} \frac{(1-s)^{\alpha-\mu-1} \xi_{j}^{\alpha-\mu-1}}{\Gamma\left(\alpha-\mu_{n-1}\right)} h(s) d s \\
& -\frac{t^{\alpha-\mu_{n-1}-1}}{1-\sum_{j=1}^{p-2} a_{j} \xi_{j}^{\alpha-\mu-1}} \sum_{j=1}^{p-2} a_{j} \int_{0}^{\xi_{j}} \frac{\left(\xi_{j}-s\right)^{\alpha-\mu-1}}{\Gamma\left(\alpha-\mu_{n-1}\right)} h(s) d s \\
& =\int_{0}^{1}\left(k_{1}(t, s)+\frac{t^{\alpha-\mu_{n-1}-1}}{1-\sum_{j=1}^{p-2} a_{j} \xi_{j}^{\alpha-\mu-1}} \sum_{j=1}^{p-2} a_{j} k_{2}\left(\xi_{j}, s\right)\right) h(s) d s \\
& =\int_{0}^{1} K(t, s) h(s) d s
\end{aligned}
$$

The proof is completed.

Lemma 2.4 $|K(t, s)| \leq M(1-s)^{\alpha-\mu-1}$, for $t, s \in[0,1]$, where

$$
M=\frac{1+\frac{\sum_{j=1}^{p-2} a_{j}}{\left|1-\sum_{j=1}^{p-2} a_{j} \xi_{j}^{\alpha-\mu-1}\right|}}{\Gamma\left(\alpha-\mu_{n-1}\right)} .
$$

Proof Obviously, for $t, s \in[0,1]$, we have $k_{i}(t, s) \leq \frac{(1-s)^{\alpha-\mu-1}}{\Gamma\left(\alpha-\mu_{n-1}\right)}, i=1,2$. Thus

$$
\begin{aligned}
|K(t, s)| & =\left|k_{1}(t, s)+\frac{t^{\alpha-\mu_{n-1}-1}}{1-\sum_{j=1}^{p-2} a_{j} \xi_{j}^{\alpha-\mu-1}} \sum_{j=1}^{p-2} a_{j} k_{2}\left(\xi_{j}, s\right)\right| \\
& \leq \frac{(1-s)^{\alpha-\mu-1}}{\Gamma\left(\alpha-\mu_{n-1}\right)}+\frac{\sum_{j=1}^{p-2} a_{j}(1-s)^{\alpha-\mu-1}}{\Gamma\left(\alpha-\mu_{n-1}\right) \mid 1-\sum_{j=1}^{p-2} a_{j} \xi_{j}^{\alpha-\mu-1}} \mid
\end{aligned}
$$




$$
\leq\left(1+\frac{\sum_{j=1}^{p-2} a_{j}}{\left|1-\sum_{j=1}^{p-2} a_{j} \xi_{j}^{\alpha-\mu-1}\right|}\right) \frac{(1-s)^{\alpha-\mu-1}}{\Gamma\left(\alpha-\mu_{n-1}\right)}
$$

This completes the proof.

Now let us consider the following modified problem of BVP (1.1)

$$
\left\{\begin{array}{l}
-\mathscr{D}^{\alpha-\mu_{n-1}} v(t)=f\left(t, I^{\mu_{n-1}} v(t), I^{\mu_{n-1}-\mu_{1}} v(t), \ldots, I^{\mu_{n-1}-\mu_{n-2}} v(t), v(t)\right) \\
v(0)=0, \quad \mathbb{D}^{\mu-\mu_{n-1}} v(1)=\sum_{j=1}^{p-2} a_{j} \mathscr{D}^{\mu-\mu_{n-1}} v\left(\xi_{j}\right)
\end{array}\right.
$$

Lemma 2.5 Let $x(t)=I^{\mu_{n-1}} v(t), v(t) \in C[0,1]$. Then (2.9) can be transformed into (1.1). Moreover, if $v \in C([0,1], \mathbb{R})$ is a solution of the problem (2.9), then the function $x(t)=$ $I^{\mu_{n-1}} v(t)$ is a solution of the problem (1.1).

Proof Substituting $x(t)=I^{\mu_{n-1}} v(t)$ into (1.1), by Lemmas 2.1 and 2.2, we can obtain that

$$
\begin{aligned}
& \mathbb{D}^{\alpha} x(t)=\frac{d^{n}}{d t^{n}} I^{n-\alpha} x(t)=\frac{d^{n}}{d t^{n}} I^{n-\alpha} I^{\mu_{n-1}} v(t) \\
& =\frac{d^{n}}{d t^{n}} I^{n-\alpha+\mu_{n-1}} v(t)=\mathfrak{D}^{\alpha-\mu_{n-1}} v(t), \\
& \mathscr{D}^{\mu_{1}} x(t)=\mathscr{D}^{\mu_{1}} I^{\mu_{n-1}} v(t)=I^{\mu_{n-1}-\mu_{1}} v(t), \\
& \mathscr{D}^{\mu_{2}} x(t)=\mathscr{D}^{\mu_{2}} I^{\mu_{n-1}} v(t)=I^{\mu_{n-1}-\mu_{2}} v(t), \\
& \mathscr{D}^{\mu_{n-2}} x(t)=\mathscr{D}^{\mu_{n-2}} I^{\mu_{n-1}} v(t)=I^{\mu_{n-1}-\mu_{n-2}} v(t), \\
& \mathscr{D}^{\mu_{n-1}} x(t)=\mathscr{D}^{\mu_{n-1}} I^{\mu_{n-1}} v(t)=v(t),
\end{aligned}
$$

and also $\mathscr{D}^{\mu_{n-1}} x(0)=v(0)=0$. It follows from $\mathscr{D}^{\mu} x(t)=\mathscr{D}^{\mu} I^{\mu_{n-1}} v(t)=\frac{d^{n}}{d t^{n}} I^{n-\mu} I^{\mu_{n-1}} v(t)=$ $\mathscr{D}^{\mu-\mu_{n-1}} v(t)$ that $\mathscr{D}^{\mu-\mu_{n-1}} v(1)=\sum_{j=1}^{p-2} a_{j} \mathscr{D}^{\mu-\mu_{n-1}} v\left(\xi_{j}\right)$. Using $x(t)=I^{\mu_{n-1}} v(t), v \in C[0,1]$, (2.9) is transformed into (1.1).

Now, let $v \in C([0,1], \mathbb{R})$ be a solution for the problem (2.9). Then, from Lemma 2.1, (2.9) and (2.10), one has

$$
\begin{aligned}
-\mathscr{D}^{\alpha} x(t) & =-\frac{d^{n}}{d t^{n}} I^{n-\alpha} x(t)=-\frac{d^{n}}{d t^{n}} I^{n-\alpha} I^{\mu_{n-1}} v(t) \\
& =-\frac{d^{n}}{d t^{n}} I^{n-\alpha+\mu_{n-1}} v(t)=-\mathfrak{D}^{\alpha-\mu_{n-1}} v(t) \\
& =f\left(t, I^{\mu_{n-1}} v(t), I^{\mu_{n-1}-\mu_{1}} v(t), \ldots, I^{\mu_{n-1}-\mu_{n-2}} v(t), v(t)\right) \\
& =f\left(t, x(t), \mathbb{D}^{\mu_{1}} x(t), \mathbb{D}^{\mu_{2}} x(t), \ldots, \mathbb{D}^{\mu_{n-1}} x(t)\right), \quad 0<t<1 .
\end{aligned}
$$

Notice

$$
I^{\alpha} v(t)=\frac{1}{\Gamma(\alpha)} \int_{0}^{t}(t-s)^{\alpha-1} v(s) d s
$$


which implies that $I^{\alpha} v(0)=0$. Thus from (2.10), for $i=1,2, \ldots, n-1$, we have

$$
x(0)=0, \quad \mathbb{D}^{\mu_{i}} x(0)=0, \quad \boldsymbol{D}^{\mu} x(1)=\sum_{j=1}^{p-2} a_{j} \mathfrak{D}^{\mu} x\left(\xi_{j}\right) .
$$

Moreover, it follows from the monotonicity and property of $I^{\mu_{n-1}}$ that

$$
I^{\mu_{n-1}} v \in C([0,1],[0,+\infty)) .
$$

Consequently, $x(t)=I^{\mu_{n-1}} v(t)$ is a solution of the problem (1.1).

Now let us define an operator $T: C[0,1] \rightarrow C[0,1]$ by

$$
(T v)(t)=\int_{0}^{1} K(t, s) f\left(s, I^{\mu_{n-1}} v(s), I^{\mu_{n-1}-\mu_{1}} v(s), \ldots, I^{\mu_{n-1}-\mu_{n-2}} v(s), v(s)\right) d s .
$$

Clearly, the fixed point of the operator $T$ is a solution of BVP (2.9); and consequently is also a solution of BVP (1.1) from Lemma 2.5.

Lemma 2.6 $T: C[0,1] \rightarrow C[0,1]$ is a completely continuous operator.

Proof Noticing that $f:[0,1] \times \mathbb{R}^{n} \rightarrow \mathbb{R}$ is continuous, by using the Ascoli-Arzela theorem and standard arguments, the result can easily be shown.

Lemma 2.7 (see [25]) Let $X$ be a real Banach space, $\Omega$ be a bounded open subset of $X$, where $\theta \in \Omega, T: \bar{\Omega} \rightarrow X$ is a completely continuous operator. Then, either there exists $x \in$ $\partial \Omega, \lambda>1$ such that $T(x)=\lambda x$, or there exists a fixed point $x^{*} \in \bar{\Omega}$.

\section{Main results}

For the convenience of expression in rest of the paper, we let $\mu_{0}=0$.

Theorem 3.1 Suppose $f(t, 0, \ldots, 0) \not \equiv 0$ for any $t \in[0,1]$. Moreover, there exist nonnegative functions $p_{1}, p_{2}, \ldots, p_{n}, q \in L^{1}[0,1]$ such that

$$
\left|f\left(t, u_{1}, u_{2}, \ldots, u_{n}\right)\right| \leq \sum_{i=1}^{n} p_{i}(t)\left|u_{i}\right|+q(t), \quad \text { a.e. }\left(t, u_{1}, u_{2}, \ldots, u_{n}\right) \in[0,1] \times \mathbb{R}^{n},
$$

and

$$
M \int_{0}^{1}(1-s)^{\alpha-\mu-1} \sum_{i=1}^{n} p_{i}(s) d s<\left(1+\sum_{i=0}^{n-2} \frac{1}{\Gamma\left(\mu_{n-1}-\mu_{i}\right)}\right)^{-1}
$$

where $M$ is defined by (2.8). Then BVP (1.1) has at least one nontrivial solution.

Proof Since $f(t, 0, \ldots, 0) \not \equiv 0$, there exists $[\sigma, \tau] \in[0,1]$ such that

$$
\min _{t \in[\sigma, \tau]}|f(t, 0, \ldots, 0)|>0
$$


By condition (3.1), we have $q(t) \geq|f(t, 0, \ldots, 0)|$, a.e. $t \in[0,1]$, thus

$$
\int_{0}^{1}(1-s)^{\alpha-\mu-1} q(s) d s>0
$$

On the other hand, from (3.2), we know

$$
\left(1+\sum_{i=0}^{n-2} \frac{1}{\Gamma\left(\mu_{n-1}-\mu_{i}\right)}\right) M \int_{0}^{1}(1-s)^{\alpha-\mu-1} \sum_{i=1}^{n} p_{i}(s) d s<1 .
$$

Take

$$
r=\frac{M \int_{0}^{1}(1-s)^{\alpha-\mu-1} q(s) d s}{1-\left(1+\sum_{i=0}^{n-2} \frac{1}{\Gamma\left(\mu_{n-1}-\mu_{i}\right)}\right) M \int_{0}^{1}(1-s)^{\alpha-\mu-1} \sum_{i=1}^{n} p_{i}(s) d s}
$$

then $r>0$.

Now let $\Omega_{r}=\{v \in C[0,1]:\|x\|<r\}$, suppose $v \in \partial \Omega_{r}, \lambda>1$ such that $T v=\lambda \nu$. Then

$$
\begin{aligned}
\lambda r & =\lambda\|v\|=\|T v\|=\max _{t \in[0,1]}|T v(t)| \\
& \leq M \int_{0}^{1}(1-s)^{\alpha-\mu-1} f\left(s, I^{\mu_{n-1}} v(s), I^{\mu_{n-1}-\mu_{1}} v(s), \ldots, I^{\mu_{n-1}-\mu_{n-2}} v(s), v(s)\right) d s .
\end{aligned}
$$

Moreover, for $i=0,1,2, \ldots, n-2$,

$$
\left|I^{\mu_{n-1}-\mu_{i}} v(t)\right|=\left|\int_{0}^{t} \frac{(t-s)^{\mu_{n-1}-\mu_{i}-1} v(s)}{\Gamma\left(\mu_{n-1}-\mu_{i}\right)} d s\right| \leq \frac{\|v\|}{\Gamma\left(\mu_{n-1}-\mu_{i}\right)},
$$

thus we have, by hypothesis (3.1),

$$
\begin{aligned}
& \left|f\left(s, I^{\mu_{n-1}} v(s), I^{\mu_{n-1}-\mu_{1}} v(s), \ldots, I^{\mu_{n-1}-\mu_{n-2}} v(s), v(s)\right)\right| \\
& \leq p_{1}(s)\left|I^{\mu_{n-1}} v(s)\right|+p_{2}(s)\left|I^{\mu_{n-1}-\mu_{1}} v(s)\right|+\cdots \\
& \quad+p_{n-1}(s)\left|I^{\mu_{n-1}-\mu_{n-2}} v(s)\right|+p_{n}(s)|v(s)|+q(s) \\
& \leq \frac{\|v\|}{\Gamma\left(\mu_{n-1}\right)} p_{1}(s)+\frac{\|v\|}{\Gamma\left(\mu_{n-1}-\mu_{1}\right)} p_{2}(s)+\cdots \\
& \quad+\frac{\|v\|}{\Gamma\left(\mu_{n-1}-\mu_{n-2}\right)} p_{n-1}(s)+\|v\| p_{n}(s)+q(s) \\
& \leq\left(1+\sum_{i=0}^{n-2} \frac{1}{\Gamma\left(\mu_{n-1}-\mu_{i}\right)}\right)\|v\|\left[p_{1}(s)+p_{2}(s)+\cdots+p_{n-1}(s)+p_{n}(s)\right]+q(s)
\end{aligned}
$$

Consequently, from (3.3), we have

$$
\begin{aligned}
\lambda r \leq & \left(1+\sum_{i=0}^{n-2} \frac{1}{\Gamma\left(\mu_{n-1}-\mu_{i}\right)}\right) M \int_{0}^{1}(1-s)^{\alpha-\mu-1} \sum_{i=1}^{n} p_{i}(s) d s\|v\| \\
& +M \int_{0}^{1}(1-s)^{\alpha-\mu-1} q(s) d s
\end{aligned}
$$




$$
=r\left(1+\sum_{i=0}^{n-2} \frac{1}{\Gamma\left(\mu_{n-1}-\mu_{i}\right)}\right) M \int_{0}^{1}(1-s)^{\alpha-\mu-1} \sum_{i=1}^{n} p_{i}(s) d s+M \int_{0}^{1}(1-s)^{\alpha-\mu-1} q(s) d s .
$$

Therefore,

$$
\begin{aligned}
\lambda & \leq\left(1+\sum_{i=0}^{n-2} \frac{1}{\Gamma\left(\mu_{n-1}-\mu_{i}\right)}\right) M \int_{0}^{1}(1-s)^{\alpha-\mu-1} \sum_{i=1}^{n} p_{i}(s) d s+\frac{M \int_{0}^{1}(1-s)^{\alpha-\mu-1} q(s) d s}{r} \\
& =1 .
\end{aligned}
$$

This contradicts $\lambda>1$. By Lemma 2.7, $T$ has a fixed point $v^{*} \in \bar{\Omega}$, since $f(t, 0, \ldots, 0) \not \equiv 0$; so then, by Lemma 2.5, BVP (1.1) has a nontrivial solution $v^{*}$. This completes the proof.

Theorem 3.2 Suppose $f(t, 0, \ldots, 0) \not \equiv 0$ for any $t \in[0,1]$. Moreover, there exist nonnegative functions $p_{1}, p_{2}, \ldots, p_{n}, q \in L^{1}[0,1]$ such that

$$
\left|f\left(t, u_{1}, u_{2}, \ldots, u_{n}\right)\right| \leq \sum_{i=1}^{n} p_{i}(t)\left|u_{i}\right|^{\sigma_{i}}+q(t), \quad \text { a.e. }\left(t, u_{1}, u_{2}, \ldots, u_{n}\right) \in[0,1] \times \mathbb{R}^{n}
$$

where $0<\sigma_{1}, \sigma_{2}, \ldots, \sigma_{n}<1$ are nonnegative constants. Then BVP (1.1) has at least one nontrivial solution.

Proof By Lemma 2.6, we know $T: C[0,1] \rightarrow C[0,1]$ is a completely continuous operator.

Let

$$
\begin{aligned}
& a=\left(1+\sum_{i=0}^{n-2} \frac{1}{\Gamma^{\sigma_{i+1}}\left(\mu_{n-1}-\mu_{i}\right)}\right) M \int_{0}^{1}(1-s)^{\alpha-\mu-1} \sum_{i=1}^{n} p_{i}(s) d s, \\
& b=M \int_{0}^{1}(1-s)^{\alpha-\mu-1} q(s) d s .
\end{aligned}
$$

Choose

$$
R \geq\left\{(n+1) b,[(n+1) a]^{\frac{1}{1-\sigma_{1}}},[(n+1) a]^{\frac{1}{1-\sigma_{2}}}, \ldots,[(n+1) a]^{\frac{1}{1-\sigma_{n}}}\right\}
$$

and define a ball $\mathfrak{M}=\{v \in C[0,1]:\|v\| \leq R, t \in[0,1]\}$. For every $v \in \mathfrak{M}$, we have

$$
\begin{aligned}
|T v(t)| & \leq \int_{0}^{1} K(t, s)\left|f\left(s, I^{\mu_{n-1}} v(s), I^{\mu_{n-1}-\mu_{1}} v(s), \ldots, I^{\mu_{n-1}-\mu_{n-2}} v(s), v(s)\right)\right| d s \\
& \leq M \int_{0}^{1}(1-s)^{\alpha-\mu-1}\left|f\left(s, I^{\mu_{n-1}} v(s), I^{\mu_{n-1}-\mu_{1}} v(s), \ldots, I^{\mu_{n-1}-\mu_{n-2}} v(s), v(s)\right)\right| d s .
\end{aligned}
$$


On the other hand, it follows from (3.4) that

$$
\begin{aligned}
\mid f(s, & \left.I^{\mu_{n-1}} v(s), I^{\mu_{n-1}-\mu_{1}} v(s), \ldots, I^{\mu_{n-1}-\mu_{n-2}} v(s), v(s)\right) \mid \\
\leq & p_{1}(s)\left|I^{\mu_{n-1}} v(s)\right|^{\sigma_{1}}+p_{2}(s)\left|I^{\mu_{n-1}-\mu_{1}} v(s)\right|^{\sigma_{2}}+\cdots \\
& +p_{n-1}(s)\left|I^{\mu_{n-1}-\mu_{n-2}} v(s)\right|^{\sigma_{n-1}}+p_{n}(s)|v(s)|^{\sigma_{n}}+q(s) \\
\leq & \frac{\|v\|^{\sigma_{1}}}{\Gamma^{\sigma_{1}}\left(\mu_{n-1}\right)} p_{1}(s)+\frac{\|v\|^{\sigma_{2}}}{\Gamma^{\sigma_{2}}\left(\mu_{n-1}-\mu_{1}\right)} p_{2}(s)+\cdots \\
& +\frac{\|v\|^{\sigma_{n-1}}}{\Gamma^{\sigma_{n-1}}\left(\mu_{n-1}-\mu_{n-2}\right)} p_{n-1}(s)+\|v\|^{\sigma_{n}} p_{n}(s)+q(s) \\
\leq & \left(\|v\|^{\sigma_{n}}+\sum_{i=0}^{n-2} \frac{\|v\|^{\sigma_{i+1}}}{\Gamma^{\sigma_{i+1}}\left(\mu_{n-1}-\mu_{i}\right)}\right)\left[p_{1}(s)+p_{2}(s)+\cdots+p_{n-1}(s)+p_{n}(s)\right]+q(s) \\
\leq & \left(1+\sum_{i=0}^{n-2} \frac{1}{\Gamma^{\sigma_{i+1}}\left(\mu_{n-1}-\mu_{i}\right)}\right) \sum_{i=1}^{n}\|v\|^{\sigma_{i}} \sum_{i=1}^{n} p_{i}(s)+q(s) .
\end{aligned}
$$

In view of (3.5), we have the following estimate:

$$
\begin{aligned}
|T \nu(t)| \leq & \left(1+\sum_{i=0}^{n-2} \frac{1}{\Gamma^{\sigma_{i+1}}\left(\mu_{n-1}-\mu_{i}\right)}\right) M \int_{0}^{1}(1-s)^{\alpha-\mu-1} \sum_{i=1}^{n} p_{i}(s) d s \sum_{i=1}^{n}\|v\|^{\sigma_{i}} \\
& +M \int_{0}^{1}(1-s)^{\alpha-\mu-1} q(s) d s \\
= & a \sum_{i=1}^{n}\|v\|^{\sigma_{i}}+b \leq \frac{n R}{n+1}+\frac{R}{n+1}=R .
\end{aligned}
$$

Therefore, $\|T v\| \leq R$. Thus we have $T: \mathfrak{M} \rightarrow \mathfrak{M}$. Hence the Schauder fixed point theorem implies the existence of a solution in $\mathfrak{M}$ for BVP (2.9). Since $f(t, 0, \ldots, 0) \not \equiv 0$, then by Lemma 2.5, BVP (1.1) has a nontrivial solution $v^{*}$. This completes the proof.

Theorem 3.3 Suppose $f(t, 0, \ldots, 0) \not \equiv 0$ for any $t \in[0,1]$. Moreover, there exist nonnegative functions $p_{1}, p_{2}, \ldots, p_{n}, q \in L^{1}[0,1]$ such that

$$
\left|f\left(t, u_{1}, u_{2}, \ldots, u_{n}\right)\right| \leq \sum_{i=1}^{n} p_{i}(t)\left|u_{i}\right|^{\sigma_{i}}+q(t), \quad \text { a.e. }\left(t, u_{1}, u_{2}, \ldots, u_{n}\right) \in[0,1] \times \mathbb{R}^{n}
$$

where $\sigma_{1}, \sigma_{2}, \ldots, \sigma_{n}>1$ are nonnegative constants. Then BVP(1.1) has at least one nontrivial solution.

Proof The proof is similar to that of Theorem 3.2, so it is omitted.

Remark 3.1 In [13], the authors studied the cases $1<\alpha \leq 2, \mu_{1}=\mu_{2}=\cdots=\mu_{n-1}=\beta$, $0<\beta<1$, but the case of $\sigma_{i}=1, i=1,2, \ldots, n$ was not considered. Here we extend the results of [13] and fill the case $\sigma_{i}=1, i=1,2, \ldots, n$. 
Theorem 3.4 Suppose $f(t, 0, \ldots, 0) \not \equiv 0$ for any $t \in[0,1]$. Moreover, there exist nonnegative functions $p_{1}, p_{2}, \ldots, p_{n} \in L^{1}[0,1]$ such that

$$
\begin{gathered}
\left|f\left(t, u_{1}, u_{2}, \ldots, u_{n}\right)-f\left(t, v_{1}, v_{2}, \ldots, v_{n}\right)\right| \leq \sum_{i=1}^{n} p_{i}(t)\left|u_{i}-v_{i}\right| \\
\text { a.e. }\left(t, u_{1}, u_{2}, \ldots, u_{n}\right),\left(t, v_{1}, v_{2}, \ldots, v_{n}\right) \in[0,1] \times \mathbb{R}^{n}
\end{gathered}
$$

and (3.2) holds. Then BVP (1.1) has a unique nontrivial solution.

Proof In fact, if $v_{1}=v_{2}=\cdots=v_{n} \equiv 0$, then we have

$$
\left|f\left(t, u_{1}, u_{2}, \ldots, u_{n}\right)\right| \leq \sum_{i=1}^{n} p_{i}(t)\left|u_{i}\right|+|f(t, 0,0, \ldots, 0)| .
$$

From Theorem 3.1, we know BVP (1.1) has a nontrivial solution.

But in this case, we prefer to concentrate on the uniqueness of a nontrivial solution for BVP (1.1). Let $T$ be given in (2.11), we shall show that $T$ is a contraction. In fact, by (3.7), a similar method to Theorem 3.1, we have

$$
\begin{aligned}
& \mid f\left(s, I^{\mu_{n-1}} u(s), I^{\mu_{n-1}-\mu_{1}} u(s), \ldots, I^{\mu_{n-1}-\mu_{n-2}} u(s), u(s)\right) \\
& \quad-f\left(s, I^{\mu_{n-1}} v(s), I^{\mu_{n-1}-\mu_{1}} v(s), \ldots, I^{\mu_{n-1}-\mu_{n-2}} v(s), v(s)\right) \mid \\
& \leq\left(1+\sum_{i=0}^{n-2} \frac{1}{\Gamma\left(\mu_{n-1}-\mu_{i}\right)}\right)\|u-v\| \sum_{i=1}^{n} p_{i}(s) .
\end{aligned}
$$

And then

$$
\begin{aligned}
\|T u-T v\| \leq & M \int_{0}^{1}(1-s)^{\alpha-\mu-1} \mid f\left(s, I^{\mu_{n-1}} u(s), I^{\mu_{n-1}-\mu_{1}} u(s), \ldots, I^{\mu_{n-1}-\mu_{n-2}} u(s), u(s)\right) \\
& -f\left(s, I^{\mu_{n-1}} v(s), I^{\mu_{n-1}-\mu_{1}} v(s), \ldots, I^{\mu_{n-1}-\mu_{n-2}} v(s), v(s)\right) \mid d s \\
\leq & \left(1+\sum_{i=0}^{n-2} \frac{1}{\Gamma\left(\mu_{n-1}-\mu_{i}\right)}\right) M \int_{0}^{1}(1-s)^{\alpha-\mu-1} \sum_{i=1}^{n} p_{i}(s) d s\|u-v\| .
\end{aligned}
$$

Then (3.2) implies that $T$ is indeed a contraction. Finally, we use the Banach fixed point theorem to deduce the existence of a unique nontrivial solution to BVP (1.1).

Corollary 3.1 Suppose $f(t, 0, \ldots, 0) \not \equiv 0$ for any $t \in[0,1]$, and (3.1) holds. Then BVP (1.1) has at least one nontrivial solution if one of the following conditions holds

(1) There exists a constant $p>1$ such that

$$
\int_{0}^{1}\left[\sum_{i=1}^{n} p_{i}(s)\right]^{p} d s<\left(\frac{p(\alpha-\mu-1)}{p-1}+1\right)^{p-1}\left(M+\sum_{i=0}^{n-2} \frac{M}{\Gamma\left(\mu_{n-1}-\mu_{i}\right)}\right)^{-p} .
$$

(2) There exists a constant $\lambda>-1$ such that

$$
\sum_{i=1}^{n} p_{i}(s)<\frac{\Gamma(\alpha+\lambda-\mu-1)}{\Gamma(\alpha-\mu) \Gamma(\lambda+1)}\left(M+\sum_{i=0}^{n-2} \frac{M}{\Gamma\left(\mu_{n-1}-\mu_{i}\right)}\right)^{-1} s^{\lambda} .
$$


(3) There exists a constant $\lambda>-1$ such that

$$
\sum_{i=1}^{n} p_{i}(s)<(\alpha+\lambda-\mu)\left(M+\sum_{i=0}^{n-2} \frac{M}{\Gamma\left(\mu_{n-1}-\mu_{i}\right)}\right)^{-1}(1-s)^{\lambda} .
$$

(4) $p_{i}(s)(i=1,2, \ldots, n)$ satisfy

$$
\sum_{i=1}^{n} p_{i}(s)<(\alpha-\mu)\left(M+\sum_{i=0}^{n-2} \frac{M}{\Gamma\left(\mu_{n-1}-\mu_{i}\right)}\right)^{-1} .
$$

Proof Let

$$
R=M \int_{0}^{1}(1-s)^{\alpha-\mu-1} \sum_{i=1}^{n} p_{i}(s) d s .
$$

From the proof of Theorem 3.1, we only need to prove

$$
R<\left(1+\sum_{i=0}^{n-2} \frac{1}{\Gamma\left(\mu_{n-1}-\mu_{i}\right)}\right)^{-1}
$$

(1) If (3.8) holds, let $\frac{1}{p}+\frac{1}{q}=1$, and by using Hölder inequality,

$$
\begin{aligned}
R & \leq M\left(\int_{0}^{1}\left[\sum_{i=1}^{n} p_{i}(s)\right]^{p} d s\right)^{\frac{1}{p}}\left(\int_{0}^{1}(1-s)^{q(\alpha-\mu-1)} d s\right)^{\frac{1}{q}} \\
& =M[q(\alpha-\mu-1)+1]^{-\frac{1}{q}}\left(\int_{0}^{1}\left[\sum_{i=1}^{n} p_{i}(s)\right]^{p} d s\right)^{\frac{1}{p}} \\
& =M\left[\frac{p(\alpha-\mu-1)}{p-1}+1\right]^{-\frac{p-1}{p}}\left(\int_{0}^{1}\left[\sum_{i=1}^{n} p_{i}(s)\right]^{p} d s\right)^{\frac{1}{p}} \\
& <\left(1+\sum_{i=0}^{n-2} \frac{1}{\Gamma\left(\mu_{n-1}-\mu_{i}\right)}\right)^{-1} .
\end{aligned}
$$

(2) In this case, it follows from (3.9) that

$$
\begin{aligned}
R & <M \frac{\Gamma(\alpha+\lambda-\mu-1)}{\Gamma(\alpha-\mu) \Gamma(\lambda+1)}\left(M+\sum_{i=0}^{n-2} \frac{M}{\Gamma\left(\mu_{n-1}-\mu_{i}\right)}\right)^{-1} \int_{0}^{1}(1-s)^{\alpha-\mu-1} s^{\lambda} d s \\
& =\left(1+\sum_{i=0}^{n-2} \frac{1}{\Gamma\left(\mu_{n-1}-\mu_{i}\right)}\right)^{-1} .
\end{aligned}
$$

(3) In this case, it follows from (3.10) that

$$
R<M(\alpha+\lambda-\mu)\left(M+\sum_{i=0}^{n-2} \frac{M}{\Gamma\left(\mu_{n-1}-\mu_{i}\right)}\right)^{-1} \int_{0}^{1}(1-s)^{\alpha-\mu-1}(1-s)^{\lambda} d s
$$


Ja et al. Boundary Value Problems 2012, 2012:70

Page 13 of 16

http://www.boundaryvalueproblems.com/content/2012/1/70

$$
=\left(1+\sum_{i=0}^{n-2} \frac{1}{\Gamma\left(\mu_{n-1}-\mu_{i}\right)}\right)^{-1} .
$$

(4) If (3.11) is satisfied, we have

$$
\begin{aligned}
R & <M(\alpha-\mu)\left(M+\sum_{i=0}^{n-2} \frac{M}{\Gamma\left(\mu_{n-1}-\mu_{i}\right)}\right)^{-1} \int_{0}^{1}(1-s)^{\alpha-\mu-1} d s \\
& =\left(1+\sum_{i=0}^{n-2} \frac{1}{\Gamma\left(\mu_{n-1}-\mu_{i}\right)}\right)^{-1} .
\end{aligned}
$$

This completes the proof of Corollary 3.1.

Corollary 3.2 Suppose $f(t, 0, \ldots, 0) \not \equiv 0$ for any $t \in[0,1]$. Moreover,

$$
0 \leq \limsup _{\sum_{i=1}^{n}\left|u_{i}\right| \rightarrow+\infty} \max _{t \in[0,1]} \frac{\left|f\left(t, u_{1}, u_{2}, \ldots, u_{n}\right)\right|}{\sum_{i=1}^{n}\left|u_{i}\right|}<\frac{\alpha-\mu}{M}\left(1+\sum_{i=0}^{n-2} \frac{1}{\Gamma\left(\mu_{n-1}-\mu_{i}\right)}\right)^{-1} .
$$

Then BVP (1.1) has at least one nontrivial solution.

Proof Take $\epsilon>0$ such that

$$
\frac{\alpha-\mu}{M}\left(1+\sum_{i=0}^{n-2} \frac{1}{\Gamma\left(\mu_{n-1}-\mu_{i}\right)}\right)^{-1}-\epsilon>0
$$

by (3.12), there exists a large enough constant $R_{0}>0$ such that for any $t \in[0,1], \sum_{i=1}^{n}\left|u_{i}\right| \geq$ $R_{0}$, one has

$$
\left|f\left(t, u_{1}, u_{2}, \ldots, u_{n}\right)\right| \leq\left(\frac{\alpha-\mu}{M}\left(1+\sum_{i=0}^{n-2} \frac{1}{\Gamma\left(\mu_{n-1}-\mu_{i}\right)}\right)^{-1}-\epsilon\right) \sum_{i=1}^{n}\left|u_{i}\right| .
$$

Let

$$
\hbar=\max _{t \in[0,1], \sum_{i=1}^{n}\left|u_{i}\right| \leq R_{0}}\left|f\left(t, u_{1}, u_{2}, \ldots, u_{n}\right)\right| .
$$

Then for any $\left(t, u_{1}, u_{2}, \ldots, u_{n}\right) \in[0,1] \times \mathbb{R}^{n}$, we have

$$
\left|f\left(t, u_{1}, u_{2}, \ldots, u_{n}\right)\right| \leq \hbar+\left(\frac{\alpha-\mu}{M}\left(1+\sum_{i=0}^{n-2} \frac{1}{\Gamma\left(\mu_{n-1}-\mu_{i}\right)}\right)^{-1}-\epsilon\right) \sum_{i=1}^{n}\left|u_{i}\right| .
$$

Let

$$
\sum_{i=1}^{n} p_{i}(s)=\left(\frac{\alpha-\mu}{M}\left(1+\sum_{i=0}^{n-2} \frac{1}{\Gamma\left(\mu_{n-1}-\mu_{i}\right)}\right)^{-1}-\epsilon\right), \quad q(s)=\hbar,
$$

we prove

$$
R<\left(1+\sum_{i=0}^{n-2} \frac{1}{\Gamma\left(\mu_{n-1}-\mu_{i}\right)}\right)^{-1} .
$$


In fact,

$$
\begin{aligned}
R & =M \int_{0}^{1}(1-s)^{\alpha-\mu-1} \sum_{i=1}^{n} p_{i}(s) d s \\
& \leq M\left(\frac{\alpha-\mu}{M}\left(1+\sum_{i=0}^{n-2} \frac{1}{\Gamma\left(\mu_{n-1}-\mu_{i}\right)}\right)^{-1}-\epsilon\right) \int_{0}^{1}(1-s)^{\alpha-\mu-1} d s \\
& <\left(1+\sum_{i=0}^{n-2} \frac{1}{\Gamma\left(\mu_{n-1}-\mu_{i}\right)}\right)^{-1} .
\end{aligned}
$$

Then it follows from Theorem 3.1 that BVP (1.1) has at least one nontrivial solution.

\section{Examples}

Example 4.1 Consider the boundary value problem

$$
\left\{\begin{aligned}
&-D^{\frac{5}{2}} x(t)= \frac{t \sin x(t)}{100 \sqrt{\pi+|x(t)|}}-\frac{\mathfrak{D}^{\frac{9}{8}} x(t)}{10 \sqrt{2+\left|D^{\frac{5}{4}} x(t)\right|}} \\
&+\frac{\left(1+t^{2}\right) \mathfrak{D}^{\frac{5}{4}} x(t)}{100}+t^{\frac{3}{2}}+\cos t, \quad t \in(0,1), \\
& x(0)=\mathfrak{D}^{\frac{9}{8}} x(0)=\mathfrak{D}^{\frac{5}{4}} x(0)=0, \quad \boldsymbol{D}^{\frac{11}{8}} x(1)=\frac{\sqrt{2}}{2} \boldsymbol{D}^{\frac{11}{8}}\left(\frac{1}{4}\right)+\frac{1}{5} \boldsymbol{D}^{\frac{11}{8}}\left(\frac{1}{2}\right) .
\end{aligned}\right.
$$

Proof Let $\alpha=\frac{5}{2}, \mu_{1}=\frac{9}{8}, \mu_{2}=\frac{5}{4}, \mu=\frac{11}{8}$, and set

$$
\begin{aligned}
& f\left(t, u_{1}, u_{2}, u_{3}\right)=\frac{t \sin u_{1}}{100 \sqrt{\pi+\left|u_{1}\right|}}-\frac{u_{2}}{10 \sqrt{2+\left|u_{3}\right|}}+\frac{\left(1+t^{2}\right) u_{3}}{100}+t^{\frac{3}{2}}+\cos t, \\
& p_{1}(t)=\frac{t}{100 \sqrt{\pi}}, \quad p_{2}(t)=\frac{1}{10 \sqrt{2}}, \quad p_{3}(t)=\frac{1+t^{2}}{100}, \quad q(t)=t^{\frac{3}{2}}+\cos t .
\end{aligned}
$$

Then

$$
\left|f\left(t, u_{1}, u_{2}, u_{3}\right)\right| \leq p_{1}(t)\left|u_{1}\right|+p_{2}(t)\left|u_{2}\right|+p_{3}(t)\left|u_{3}\right|+q(t),
$$

and

$$
\begin{aligned}
& \left(1+\sum_{i=0}^{n-2} \frac{1}{\Gamma\left(\mu_{n-1}-\mu_{i}\right)}\right)^{-1}=\left(\frac{1}{\Gamma\left(\frac{5}{4}\right)}+\frac{1}{\Gamma\left(\frac{1}{8}\right)}+1\right)^{-1} \approx 0.4472, \\
& M=\frac{1+\frac{\sum_{j=1}^{p-2} a_{j}}{\left|1-\sum_{j=1}^{p-2} a_{j} \xi_{j}^{\alpha-\mu-1}\right|}}{\Gamma\left(\alpha-\mu_{n-1}\right)} \approx 2.3934 .
\end{aligned}
$$

Thus we have

$$
\begin{aligned}
M \int_{0}^{1}(1-s)^{\alpha-\mu-1} \sum_{i=1}^{n} p_{i}(s) d s & =2.3934 \int_{0}^{1}(1-s)^{\alpha-\mu-1} \sum_{i=1}^{n} p_{i}(s) d s \\
& \approx 0.07679 \times 2.3934 \approx 0.1838<0.4472 .
\end{aligned}
$$


Thus the condition (3.2) in Theorem 3.1 is satisfied, and from Theorem 3.1, BVP (4.1) has a nontrivial solution.

Example 4.2 Consider the boundary value problem

$$
\left\{\begin{aligned}
-\mathscr{D}^{\frac{8}{3}} x(t)= & \frac{1}{2}\left(t-t^{2}\right) x^{5}(t)-\left(\sin t+e^{t}\right)\left[\mathscr{D}^{\frac{7}{6}} x(t)\right]^{\frac{9}{8}} \\
& +2 t^{3}\left[\mathscr{D}^{\frac{4}{3}} x(t)\right]^{3}+e^{t}+\sqrt{t}, \quad t \in(0,1), \\
x(0)=\mathscr{D}^{\frac{7}{6}} x(0)=\mathscr{D}^{\frac{4}{3}} x(0)=0, & t \\
\mathscr{D}^{\frac{3}{2}} x(1)= & \frac{1}{\pi} \mathscr{D}^{\frac{3}{2}}\left(\frac{1}{3}\right)-2 \mathscr{D}^{\frac{3}{2}}\left(\frac{2}{3}\right)+\frac{1}{2} \mathscr{D}^{\frac{3}{2}}\left(\frac{3}{4}\right) .
\end{aligned}\right.
$$

Proof Let

$$
\begin{aligned}
& f\left(t, u_{1}, u_{2}, u_{3}\right)=\frac{1}{2}\left(t-t^{2}\right)\left|u_{1}\right|^{5}+\left(\sin t+e^{t}\right)\left|u_{2}\right|^{\frac{9}{8}}+2 t^{3}\left|u_{3}\right|^{3}+e^{t}+\sqrt{t} \\
& p_{1}(t)=\frac{1}{2}\left(t-t^{2}\right), \quad p_{2}(t)=\sin t+e^{t}, \quad p_{3}(t)=2 t^{3}, \quad q(t)=e^{t}+\sqrt{t}
\end{aligned}
$$

Then

$$
\left|f\left(t, u_{1}, u_{2}, u_{3}\right)\right| \leq p_{1}(t)\left|u_{1}\right|^{5}+p_{2}(t)\left|u_{2}\right|^{\frac{9}{8}}+p_{3}(t)\left|u_{3}\right|^{3}+q(t), \quad t \in[0,1]
$$

Thus Theorem 3.4 guarantees a nontrivial solution for BVP (4.2).

\section{Competing interests}

The authors declare that they have no competing interests.

\section{Authors' contributions}

The work presented here was carried out in collaboration between all authors. Each of the authors contributed to every part of this study equally and read and approved the final version of the manuscript.

\section{Acknowledgement}

The authors thank the referee for helpful comments and suggestions which led to an improvement of the paper. The authors were supported financially by the National Natural Science Foundation of China (11071141) and the Natural Science Foundation of Shandong Province of China (ZR2010AM017) and the Fundamental Research Funds for the Central Universities (Grant No. HIT. NSRIF. 2010091), the National Science Foundation for Post-doctoral Scientists of China (Grant No. 2012M510956).

\section{Received: 7 April 2012 Accepted: 15 June 2012 Published: 3 July 2012}

\section{References}

1. Diethelm, K, Freed, AD: On the solutions of nonlinear fractional order differential equations used in the modelling of viscoplasticity. In: Keil, F, Mackens, W, Voss, H, Werthers, J (eds.) Scientific Computing in Chemical Engineering II Computational Fluid Dynamics, Reaction Engineering and Molecular Properties. Springer, Heidelberg (1999)

2. Gaul, L, Klein, P, Kempffe, S: Damping description involving fractional operators. Mech. Syst. Signal Process. 5, 81-88 (1991)

3. Glockle, WG, Nonnenmacher, TF: A fractional calculus approach of self-similar protein dynamics. Biophys. J. 68, 46-53 (1995)

4. Mainardi, F: Fractional calculus: some basic problems in continuum and statistical mechanics. In: Carpinteri, CA, Mainardi, F (eds.) Fractal and Fractional Calculus in Continuum Mechanics. Springer, Vienna (1997)

5. Metzler, F, Schick, W, Kilian, HG, Nonnenmache, TF: Relaxation in filled polymers: a fractional calculus approach. J. Chem. Phys. 103, 7180-7186 (1995)

6. Oldham, KB, Spanier, J: The Fractional Calculus. Academic Press, New York (1974)

7. Kilbas, AA, Srivastava, HM, Trujillo, JJ: Theory and Applications of Fractional Differential Equations. North-Holland Mathematics Studies. Elsevier, Amsterdam (2006)

8. Miller, KS, Ross, B: An Introduction to the Fractional Calculus and Fractional Differential Equations. Wiley, New York (1993) 
9. Podlubny, I: Fractional Differential Equations. Mathematics in Science and Engineering. Academic Press, New York (1999)

10. Salem, $\mathrm{AH}$ : On the fractional $m$-point boundary value problem in reflexive Banach space and the weak topologies. J. Comput. Appl. Math. 224, 565-572 (2009)

11. Zhang, S: Positive solutions to singular boundary value problem for nonlinear fractional differential equation. Comput. Math. Appl. 59, 1300-1309 (2010)

12. Goodrich, CS: Existence of a positive solution to a class of fractional differential equations. Appl. Math. Lett. 23, 1050-1055 (2010)

13. Rehman, M, Khan, R: Existence and uniqueness of solutions for multi-point boundary value problems for fractional differential equations. Appl. Math. Lett. 23, 1038-1044 (2010)

14. Zhang, X, Liu, L, Wu, Y: Multiple positive solutions of a singular fractional differential equation with negatively perturbed term. Math. Comput. Model. 55, 1263-1274 (2012)

15. Bai, Z: On positive solutions of a nonlocal fractional boundary value problem. Nonlinear Anal. 72, 916-924 (2010)

16. Ahmad, B, Nieto, JJ: Riemann-Liouville fractional integro-differential equations with fractional nonlocal integral boundary conditions. Bound. Value Probl. 2011, 36 (2011)

17. Goodrich, CS: Existence of a positive solution to systems of differential equations of fractional order. Comput. Math. Appl. 62, 1251-1268 (2011)

18. Goodrich, CS: Existence and uniqueness of solutions to a fractional difference equation with nonlocal conditions. Comput. Math. Appl. 61, 191-202 (2011)

19. Goodrich, CS: Positive solutions to boundary value problems with nonlinear boundary conditions. Nonlinear Anal. 75 , 417-432 (2012)

20. Zhang, X, Han, Y: Existence and uniqueness of positive solutions for higher order nonlocal fractional differential equations. Appl. Math. Lett. 25, 555-560 (2012)

21. $\mathrm{Wu}, \mathrm{J}, \mathrm{Zhang}, \mathrm{X}, \mathrm{Liu}, \mathrm{L}, \mathrm{Wu}, \mathrm{Y}$ : Positive solutions of higher order nonlinear fractional differential equations with changing-sign measure. Adv. Differ. Equ. 2012, 71 (2012)

22. Zhang, X, Liu, L, Wiwatanapataphee, B, Wu, Y: Solutions of eigenvalue problems for a class of fractional differential equations with derivatives. Abstr. Appl. Anal. 2012, Article ID 512127 (2012)

23. $\mathrm{Wu}, \mathrm{T}$, Zhang, $\mathrm{X}$ : Solutions of sign-changing fractional differential equation with the fractional. Abstr. Appl. Anal. 2012 Article ID $797398(2012)$

24. Zhang, X, Liu, L, Wu, Y: The eigenvalue problem for a singular higher order fractional differential equation involving fractional derivatives. Appl. Math. Comput. 218, 8526-8536 (2012)

25. Deimling, K: Nonlinear Functional Analysis. Springer, Berlin (1985)

doi:10.1186/1687-2770-2012-70

Cite this article as: Jia et al.: Nontrivial solutions for a higher fractional differential equation with fractional multi-point boundary conditions. Boundary Value Problems 2012 2012:70.

\section{Submit your manuscript to a SpringerOpen ${ }^{\circ}$ journal and benefit from:}

- Convenient online submission

- Rigorous peer review

- Immediate publication on acceptance

- Open access: articles freely available online

- High visibility within the field

- Retaining the copyright to your article 